\title{
Model Based Visual Servoing Tasks with an Autonomous Humanoid Robot
}

\author{
Amine Abou Moughlbay, Enric Cervera and Philippe Martinet*
}

\begin{abstract}
Many model based techniques have been proposed in the literature for applying domestic service tasks on humanoid robots, such as teleoperation, learning from demonstration and imitation. However sensor based robot control overcomes many of the difficulties of uncertain models and unknown environments which limit the domain of application of the previous methods. Furthermore, for service and manipulation tasks, it is more suitable to study the interaction between the robot and its environment at the contact point using the sensor based control, rather than specifying the joint positions and velocities required to achieve them.

In this work we present an integration of real-time visual servoing techniques on a humanoid robot in closed loop, to perform self-localization and different manipulation tasks. Indeed, real-time model based tracking techniques are used to apply 3D visual servoing tasks on the Nao humanoid robot. The elementary tasks which are used by the robot to perform a concrete service scenario are detailed with their corresponding control laws. Finally, we present the experimental results of the following tasks: self-localization of the robot while walking, head servoing for the visibility task, detection, tracking and manipulation of environment's objects.
\end{abstract}

Amine Abou Moughlbay

Institut de Recherche en Communications et Cybernétique de Nantes, Ecole Centrale de Nantes,

1 rue de la noë, 44321 Nantes - France, e-mail: Amine.Abou-Moughlbay dec-nantes. fr

Enric Cervera

Robotic Intelligence Lab, Jaume-I University, 12071 Castello - Spain,

e-mail: Ecervera@icc.uji.es

Philippe Martinet

Institut de Recherche en Communications et Cybernétique de Nantes, Ecole Centrale de Nantes and Institut Pascal in Clermont Ferrand - France,

e-mail: Philippe.Martinet@irccyn.ec-nantes.fr

* This work has been funded by the French National Agency of Research under the reference ANR-10-SEGI-002. 


\section{Introduction}

To perform manipulation tasks, the robot interacts with its environment through contact points, such as applying forces and moments on objects. By controlling the position and velocity of these points, as well as the forces acting on them, the robot performs the desired tasks. Furthermore, in complicated and uncertain environments, end-effector motion may be subject to online modifications in order to accommodate unexpected events or to respond to sensor inputs. Thus programming of service and manipulation tasks is most conveniently accomplished by directly specifying data at the contact points (using operational space control), rather than specifying the joint positions and velocities required to achieve them (using joint space control) [1].

Many approaches have been used to apply service tasks: several works have been carried out in the area of teleoperation, by controlling a robotic system to perform tasks at a distance using a multi-modal human-system interface which provides sensory feedback to the operator and allows him to interact with the remote environment by mapping his actions. It was applied to bi-manual manipulation and walking [2], haptic interface for mobile teleoperators [3] and recently on a teleoperation system with haptic device for micro-manipulation [4].

Furthermore, many practical learning control systems are used to control complex robots involving multiple feedback sensors and multiple command variables during both repetitive and nonrepetitive operations [5]. The issue of teaching a robot to manipulate everyday objects through human demonstration has been studied by [6] who proposed a method that enables a robot to decompose a demonstrated task into sequential manipulation primitives, series of sequential rotations and translations [7]. Other earlier works used also the Model Based Control strategies, executed by automatically generating a control sequence that moves the robot to the states specified by the program to develop executives that emphasize model based approaches and deep integration of automated planning [8].

On the other hand, self-localization of service robots is one of the fundamental problems in robotics, as in many applications a robot needs to know its location in order to perform its tasks. Most of the indoor localization algorithms use particle based filters or Kalman type filters to solve the problem of noisy sensors and controls. Particle filters inherently help solve the problem of ambiguous landmarks, whereas Kalman filters must track multiple hypotheses to work in ambiguous environments [9]. In some divisions of RoboCup, algorithms are very well established, given the rich sensor data provided by laser scanners, omni-directional cameras etc. However, in more general cases, there are substantial sensor limitations particularly with the rapid motion of the camera, and the need for active perception [10]. In addition to odometry sensors, some recent alternatives use also the ambient magnetic field to control the heading of a robot in case of one-dimensional localization problem [11]. 
As previously presented, most actuated systems use sensors to obtain information about their environment. These can be a camera, ranging devices, or temperature and force sensors. Among all these feedbacks, the visual information provides the most important and instant cues for perception of the interaction with the working environment. Compared to already mentioned methods, visual servoing provides very efficient solutions to control robot motions. It supplies high positioning accuracy, good robustness to sensor noise and calibration uncertainties, and reactivity to environment changes [12].

In this work, only Sensor Based Control formalism is used to perform the desired tasks. More especially, 3D visual feedback data and Model Based Tracking (MBT) techniques are used to execute, in real-time and closed loop, many tasks on the humanoid mobile robot Nao in a semi structured environment. The robot's camera is calibrated, and a rough geometric model of the objects is available (doors, tables, pieces to grasp ...). The envisioned scenario consists of a Nao robot which can carry out service tasks, moving around the room and manipulating objects. One of the missions that can be requested from such robot would be to "Pick up the orange and green pieces and deposit them on the table nearby the door" (see Fig. 1). But to execute this mission, many problems should be addressed and resolved:

- How can the robot localize itself with respect to its environment?

- How to decompose the robot's mission into elementary tasks?

- What information should be given to the robot to execute each task?

- What are the more useful techniques the robot can use to perform these tasks?

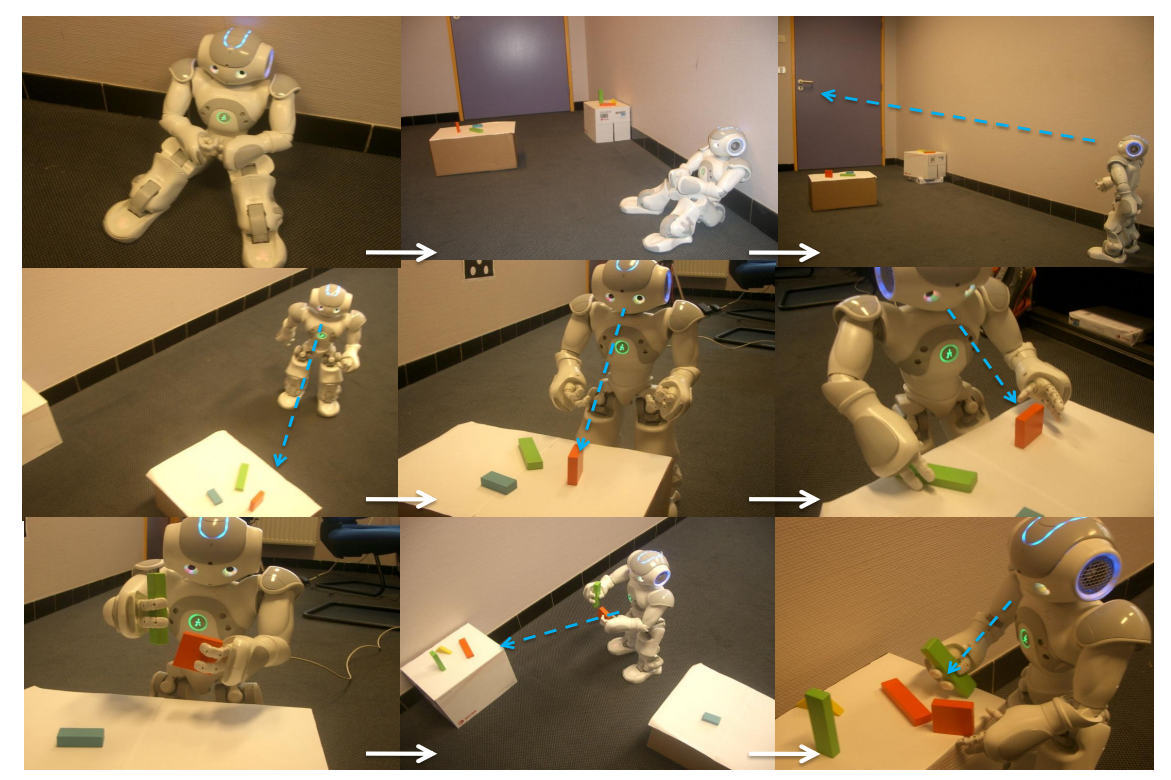

Fig. 1 Nao picks up the orange and green pieces and deposits them on the table near the door 
In the next section, we present the architecture of the Nao robot and the Model Based Tracking technique. In section 3, we define the control laws of the common elementary tasks employed during the application of the desired tasks. Implemented tasks are presented in section 4 and the experimental results in section 5 . The first part of this latter includes the localization of the robot in its environment when walking. The second one includes the simultaneous execution of object tracking, head control and manipulation by visual servoing. The final section draws some conclusions and outlines future works.

\section{System Architecture}

\subsection{Nao Architecture}

Nao Robot [13], developed by Aldebaran robotics, is a $(57 \mathrm{~cm}, 5.2 \mathrm{~kg})$ biped robot with 25 Degrees of Freedom (DOF) . It has 3-fingered robotic hands used for grasping and holding small objects (it can carry up to $300 \mathrm{~g}$ using both hands). Nao is equipped with: two ultrasound devices situated in the chest that provide space information in a range of 1 meter, two cameras situated on the top and bottom of the head, two bumpers (contact sensors on the robot's feet), a gyrometer and an accelerometer (to determine whether the robot is in a stable or unstable position).

\subsection{Visual servoing and tracking techniques}

A large variety of positioning or target tracking tasks can be implemented by controlling from one to all DOF of the system. For whatever the sensor configuration, which can vary from one camera mounted on the robot end-effector to several freestanding cameras, a set of visual features $\mathbf{s}$ has to be designed from the visual measurements obtained from the system configuration $\mathbf{x}(t)$, allowing control of the desired DOF.

A control law is thus designed so that these features $\mathbf{s}$ reach a desired value $\mathbf{s}^{*}$, defining a correct realization of the task. Indeed, if the camera velocity $\mathbf{V}_{c}$ is considered as input of the robot controller, the control law which performs the desired exponential decoupled decrease of the error $\mathbf{e}=\left(\mathbf{s}-\mathbf{s}^{*}\right)$ is given by:

$$
\mathbf{V}_{c}=-\lambda \mathbf{L}_{s}^{+}\left(\mathbf{s}-\mathbf{s}^{*}\right)
$$

where $\lambda$ is the classical proportional gain that has to be tuned to minimize the time to convergence of the task, and $\mathbf{L}_{s}^{+}$is the Moore-Penrose pseudo-inverse of the interaction matrix $\mathbf{L}_{s}$ (for more details refer to visual servo control book [14]). 


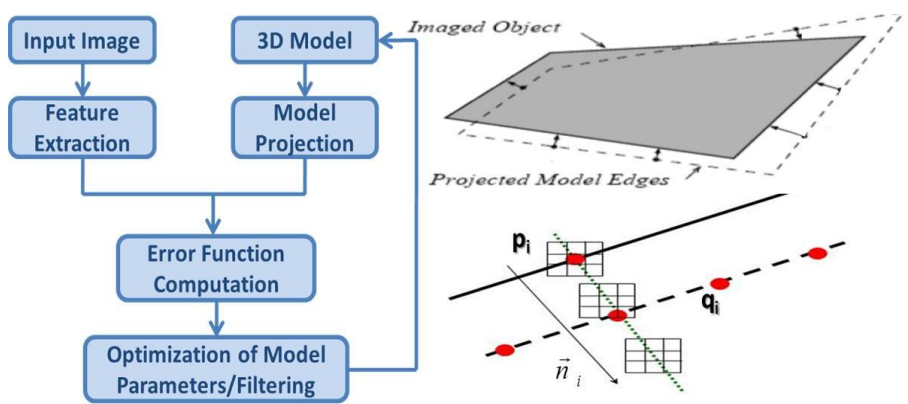

Fig. 2 Model Based tracking system (left) using the Moving edge detection technique (right)

Many tracking tools have been implemented in several visual servoing toolboxes [15]. On the Visual Servoing Platform (ViSP) [16], we find a dot tracker, a moving edges tracker, and a 3D model based tracker. The last one [17] tracks a 3D model thanks to the moving edges method, using a virtual visual servoing technique. It requires a 3D model and needs to compute the initial pose which is used to project the model on the image. The tracking method assumes that the pose corresponding to the previous image is known, the new lines are tracked, and the goal is to move the pose to match the object in the new image with the projection of the model.

The following error function (err) between image features $p_{i}$ and model projection $q_{i}$ is thus minimized along the normal direction $\mathbf{n}$ (see Fig. 2):

$$
\text { err }=\sum_{i} \Delta\left(p_{i}, q_{i}\right)=\sum_{i}\left|\left(q_{i}-p_{i}\right) \cdot\left(n_{i}\right)\right|
$$

\section{Control Law and Tasks Identification}

To execute the desired tasks, we should first define several coordinate frames on the robot's body and environment's items as represented in Fig. 3. And using the inverse kinematic model of the robot and the 3D visual servoing technique (1) detailed above, the general control law which is used to define a generic task can be written:

$$
\dot{\mathbf{q}}=-\lambda\left(\mathbf{L}_{\mathbf{s}} \mathbf{J}\right)^{+}\left(\mathbf{s}-\mathbf{s}^{*}\right)
$$

where $\dot{\mathbf{q}}$ is the robot's joint velocity and $\mathbf{J}$ is the kinematic matrix which relates camera velocity with the robot's joint velocity $\left(\mathbf{V}_{c}=\mathbf{J} \dot{\mathbf{q}}\right)$. 
Fig. 3 Useful frames in Nao's environment:

In Nao's body we consider the following frames: $\mathscr{F}_{n}$ Nao's space frame (between robot's feet), $\mathscr{F}_{t}$ on robot's torso,

$\mathscr{F}_{c}$ a camera attached frame, $\mathscr{F}_{h}$ robot's hand frame, $\mathscr{F}_{g}$ robot's gripper frame and $\mathscr{F}$ pg pre-grasping frame.

The following frames are defined in the robot's environment: $\mathscr{F}_{o}$ object's frame, $\mathscr{F}_{d}$ desired object's pose frame and $\mathscr{F}_{e}$ environment's frame for the localization task.

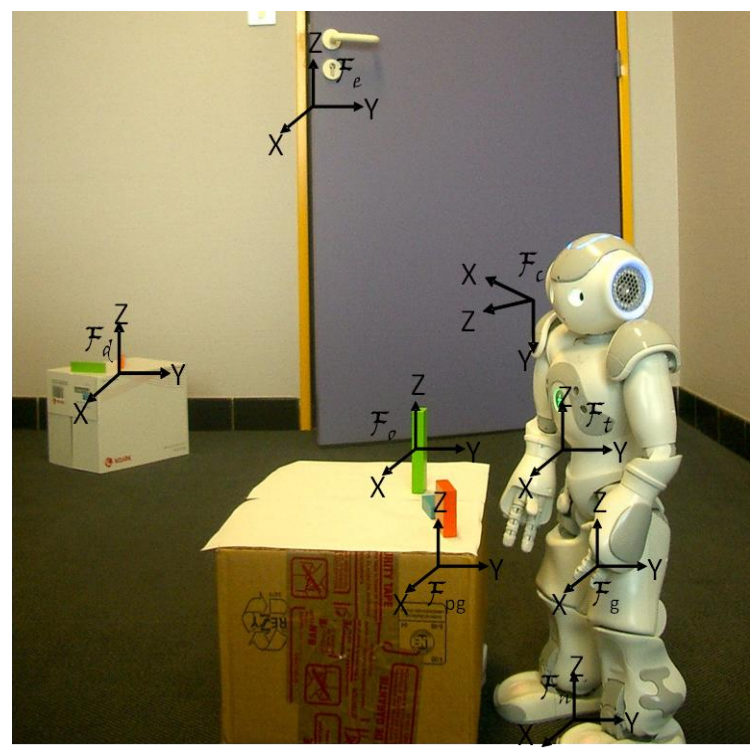

In the rest of this section, the envisaged scenario is decomposed into simpler generic tasks to reduce the complexity of the problem to be solved. Thus, the desired scenario is executed by answering the following questions:

(a) Where am I ? $\rightarrow$ Self-Localization Task

Depending on the desired mission to be executed, the robot searches for the corresponding set of models in its environment to be localized with respect to them (such as table, door, light switch, corner, ...).

After detecting the items in the field of view of the robot's camera, and choosing the nearest one to the object to manipulate $\left(\mathscr{F}_{e}\right)$, the robot calculates, using the MBT technique, its position/orientation with respect to the item in form of a homogeneous transformation matrix $\left({ }^{n} \mathbf{M}_{e}\right)$.

\section{(b) What must I do and How to do it ? $\rightarrow$ Task Scheduler}

The desired scenario is interpreted to the robot's language and decomposed into elementary tasks by the task scheduler [18]. In case of the presented scenario, the following tasks are considered:

- Localize the desired objects to manipulate

- Move in the appropriated direction

- Detect and track the desired objects

- Keep these pieces in the robot's field of view

- Move the robot's arms and grasp these pieces

- Go to the desired table and deposit objects 
(c) Which data will I use ? $\rightarrow$ Definition of used data

To execute the defined elementary tasks, the scheduler chooses the appropriate models for each one: models of the objects to manipulate, model of the environment's items (for the self-localization task), data of the robot's camera, parameters of the robot's gripper and the used items...

(d) Where to move ? $\rightarrow$ Robot Locomotion Task

This task is used to move to the region where the task should be executed. Knowing the position of at least one item of the environment $\left({ }^{n} \mathbf{M}_{e}\right)$, the robot walks in the appropriate direction of $\left(\mathscr{F}_{e}\right)$ until entering the range of a defined distance from the object.

During this task, the robot motion is controlled in real-time and closed loop to avoid some possible modifications or unexpected events. Thus an obstacle avoidance algorithm should be used to adapt the robot's trajectory [19].

(e) How to perceive ? $\rightarrow$ Detection and Tracking Tasks

Using the MBT technique of ViSP presented in 2.2, the tracker is manually initialized and the pose of the desired item is determined. Therefore it allows us to track in real-time the pose of the object to manipulate.

Furthermore, to increase its robustness, a module for automatic re-initialization of the tracker is implemented, it uses the last poses of the tracked object to estimate the actual pose. This module is used in case of a failure due to an occlusion or the fast motions of the robot's camera (especially when walking). Moreover, the automatic transition between the tracking of different objects is also implemented using the knowledge of the rough relative position between environment's objects.

(f) How to keep the concerned points in the robot's field of view ? $\rightarrow$ Visibility Task

This task consists of controlling the position/orientation of the robot's head to focus a (fixed/mobile) point of the environment (item's center, gripper, virtual point...) in the center of the camera's image.

This task can be used, for example, in hand-eye coordination for dynamic grasping of objects by focusing on the gripper-item midpoint, to keep the robot's hand and object in the robot's field of view. The used DOF in this task depends on the geometry of the robot's head and the desired complexity of the task. The head's Yaw/Pitch can be simply controlled to focus on the object's center, or a more complex task can also control the distance between the robot's head and the object.

(g) How to perform the manipulation ? $\rightarrow$ Grasping Task

This task uses the hand's control point and allows the robot to move it to a desired static/mobile pose. This task can be used to perform pre-grasping, grasping, and displacing objects tasks. 
In case of pre-grasping task, the goal position $\left({ }^{g} \mathbf{M}_{p g}\right)$ is determined using one of the grasping strategies [20]. They depend usually on the geometry of the object to manipulate and on the shape of the robot's gripper. Thus, the grasping strategy controls the relative position and/or the angle between the gripper and the item to grasp.

Likewise, for a grasping task the same technique is considered: the robot's arm moves to a desired pose by minimizing the relative distance/angle between the object and the robot's hand $\left({ }^{g} \mathbf{M}_{o}\right)$ in the Gripper's Frame.

\section{Tasks Definition}

In this part, we present preliminary results of the general scenario detailed in the previous section applied on the humanoid robot Nao. The experimented tasks are: the self-localization of the robot when walking, and the simultaneous control of robot's head and arm for tracking and grasping an object.

During the execution of the first task, only a rough model of the door and a part of the room is used, and for the second one only the approximate model of the item to grasp is given. Note that no other exteroceptive data is given from the robot's environment.

\subsection{Self-Localization Task during Locomotion}

Throughout this task, the robot tracks the door and thus it is localized with respect to the environment while walking. We use the model of the door and the lines of the room around it to initialize the MBT which gives subsequently the pose of the door in the camera's frame $\left({ }^{c} \mathbf{M}_{e}\right)$ in real-time. From the given pose value, the robot's pose can be calculated: ${ }^{e} \mathbf{M}_{n}=\left({ }^{c} \mathbf{M}_{e}\right)^{-1}\left({ }^{n} \mathbf{M}_{c}\right)^{-1}$.

Note that the tracker is automatically reinitialized each time the tracking failed due to large camera displacements during walking; it uses the last found pose of the object to reinitialize the tracking. In our experiments, the robot walks in open loop in the direction of the door for a distance of 1 meter.

\subsection{Detection and Tracking Task}

The MBT is used to track simple item models; it's initialized manually at the beginning of the application. Afterwards, the model is automatically detected and tracked; this tool allows us to determine instantly the pose of the desired item frame in the robot camera's frame $\left({ }^{c} \mathbf{M}_{o}\right)$. 


\subsection{Visibility Task}

Throughout this application, the visibility task is used for controlling the robot's head orientation to focus the item's center in the midpoint of the camera's image. Two DOF are used by this task to control the head's Yaw and Pitch. The task's goal is thus to regulate exponentially $(\dot{\mathbf{e}}=-\lambda \mathbf{e})$ the horizontal and vertical position of the center of the object projection $\mathbf{s}_{x, y}={ }^{c} \mathbf{T}_{o(x, y)}$ to zero $\left(\mathbf{s}^{*}=(0,0)\right)$.

Using the object 3D pose ${ }^{c} \mathbf{T}_{o}=(X, Y, Z)^{T}$, and the 2D pose $(x, y)^{T}$ of the tracked point (projection of 3D point in the normal image plane), we apply the control law defined in (3) using the visual primitive $\mathbf{s}=(x, y)$ and its corresponding interaction matrix $\mathbf{L}_{\mathbf{s}}$ given by:

$$
\mathbf{L}_{\mathbf{s}}=\left[\begin{array}{cccccc}
-\frac{1}{Z} & 0 & \frac{x}{Z} & x y & -\left(1+y^{2}\right) & y \\
0 & -\frac{1}{Z} & \frac{y}{Z} & 1+x^{2} & -x y & -x
\end{array}\right]
$$

Note that for this task the $\mathbf{J}$ matrix in (3) uses the Jacobian of the robot's head control point calculated from the robot's geometric model.

\subsection{Pre-Grasping Task}

During this task the robot's arm is supposed to move close to the item to grasp. According to Nao's gripper's geometry (of one DOF) and the item's shape (rectangular model), the number of constrained DOF and the pre-grasping position is predefined in the robot's manipulation parameters. This position is defined with respect to the object's position in Nao's frame ${ }^{n}\left({ }^{o} \mathbf{M}_{p g}\right)$.

In our case, regarding the gripper's and item's shapes, 4 DOF are enough to execute this task: 3 DOF constraints the gripper's pose and 1 DOF (Yaw angle) for the gripper's orientation. Furthermore, the pre-grasping distance is fixed to $5 \mathrm{~cm}$, but we should not forget that the tracker gives the item's pose in real-time, thus the desired pre-grasping pose is calculated in closed loop.

The task's target is then to move the robot's arm to the pre-grasping pose. The task's error is extracted from the relative pose between the gripper and pre-grasping point $\left({ }^{g} \mathbf{M}_{p g}\right)$ which is regulated to zero. Note that ${ }^{g} \mathbf{M}_{p g}=\left({ }^{n} \mathbf{M}_{g}\right)^{-1{ }^{n}} \mathbf{M}_{c}{ }^{c} \mathbf{M}_{o}{ }^{o} \mathbf{M}_{p g}$ where ${ }^{n} \mathbf{M}_{g}$ and ${ }^{n} \mathbf{M}_{c}$ are given by the robot's proprioceptive sensors.

Considering that the visual primitive is parameterized by $\mathbf{s}=(\mathbf{t}, \mathbf{u} \theta)$ where $\mathbf{t}$ is the position error between the current and desired frame, while $\mathbf{u} \theta$ is the orientation error, decomposed as the axis $\mathbf{u}$ and angle $\theta$ of the rotation between these two frames. The control law (3) is then applied using the Jacobian at the robot's gripper and the corresponding interaction matrix $\mathbf{L}_{\mathbf{s}}$ given by: 


$$
\mathbf{L}_{\mathbf{s}}=\left[\begin{array}{cc}
-\mathbf{I}_{3} & {[\mathbf{t}]_{\times}} \\
\mathbf{0}_{3} & \mathbf{L}_{\omega}
\end{array}\right]
$$

where $\mathbf{I}_{3}$ and $\mathbf{0}_{3}$ are the $3 \times 3$ identity and zero matrices respectively, the $\mathbf{L}_{\omega}$ matrix is given by $\mathbf{L}_{\omega}=\mathbf{I}_{3}-\frac{\theta}{2}[\mathbf{u}]_{\times}+\left(1-\frac{\operatorname{sinc}(\theta)}{\operatorname{sinc}^{2}(\theta / 2)}\right)[\mathbf{u}]_{\times}{ }^{2}$, and $[\mathbf{t}]_{\times}$is the skew symmetric matrix associated with vector $\mathbf{t}$.

\subsection{Grasping Task}

Along this task the robot's arm moves to grasp the desired item. The same number of DOF is constrained and the same technique is used to define the grasping task as in the previous case of pre-grasping, but the desired gripper pose is changed to the object's pose. Thus the task's error will be extracted from the relative pose between the gripper and the item ${ }^{g} \mathbf{M}_{o}$ which is also regulated to zero (this pose is calculated using the relation: ${ }^{g} \mathbf{M}_{o}=\left({ }^{n} \mathbf{M}_{g}\right)^{-1{ }^{n}} \mathbf{M}_{c}{ }^{c} \mathbf{M}_{o}$.

After arriving to the desired pose, the robot's gripper closes to catch the item. We should note that the visibility and pre-grasping tasks are executed in parallel, and once the pre-grasping finished, the grasping task is executed automatically.

Furthermore, a task is completed when the error norm reaches a predefined threshold value. This threshold varies with respect to the executed task: during the grasping task of small objects, a high precision is necessary unlike in the case of pre-grasping task. In our experiments, the threshold is predefined to $5 \mathrm{~mm}$ in the camera's image for the visibility task. In case of pre-grasping and grasping tasks, the precision is predefined to $5 \mathrm{~mm}$ and $1 \mathrm{~mm}$ respectively and 3 degrees for the orientation.

\section{Experimental Results}

The presented tasks in the previous section have been implemented and tested several times on the Humanoid Nao robot to ensure the efficiency of this method ${ }^{2}$. During these experiments, we used the two cameras embedded on the robot's head: the top one for localization and the bottom one for manipulation tasks. The control rate of the robot is equal to that of the camera $(20 \mathrm{~Hz})$.

\footnotetext{
${ }^{2}$ See a video of the applied tasks on Nao robot on www.youtu.be/WflA_jRBMkM
} 


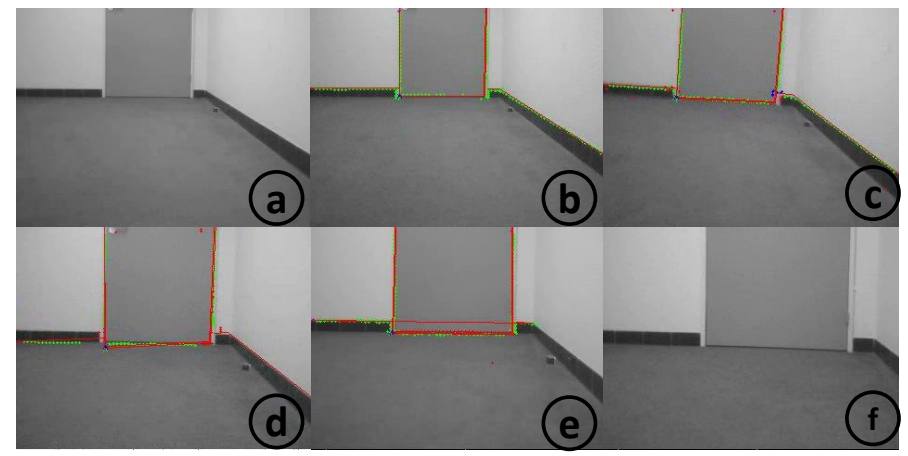

Fig. 4 Experiment photos of self-localization task during robot's locomotion

\subsection{Self-Localization Task Results}

This part corresponds to the task defined in section 4.1 above. Fig. 4-a shows the robot's environment before launching the tracking and locomotion tasks. Then Fig.4-b...e show the tracking of the door during the task's execution, and when the task is completed (Fig. 4-f).

In Fig. 5, the distance between the robot's frame $\mathscr{F}_{n}$ and the origin of the door's frame $\mathscr{F}_{e}$ is plotted, in addition to the X, Y and Z components of this pose in $\mathscr{F}_{n}$. We can notice that the distance decreases from $3.94 \mathrm{~m}$ to $2.88 \mathrm{~m}$. These results show then that the robot successfully tracks the door while walking the desired distance (1 m) with an final error of $6 \mathrm{~cm}$, which allow us to localize the robot succesfully.
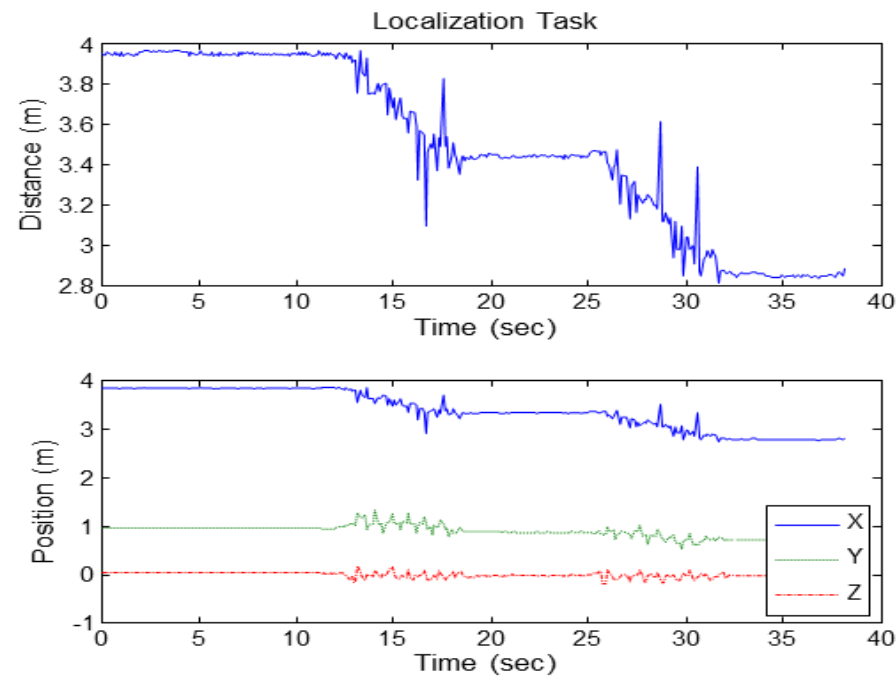

Fig. 5 Experimental results of the self-localization task in $\mathscr{F}_{n}$ frame 


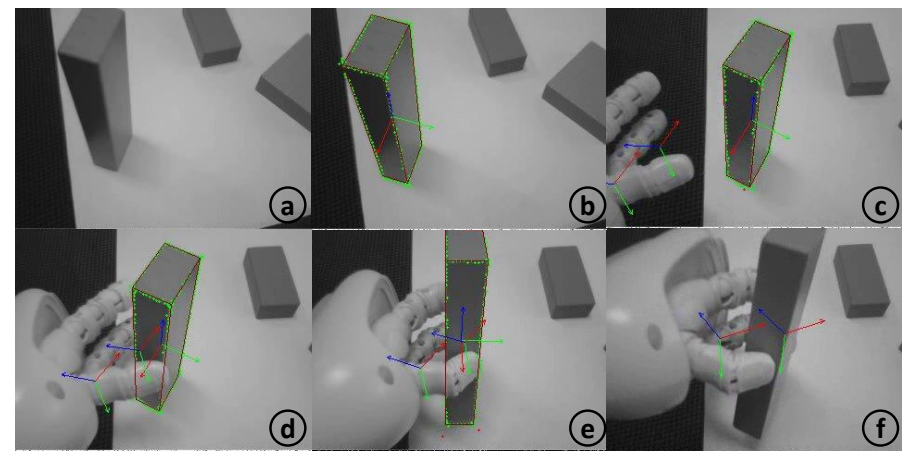

Fig. 6 Photos of tracking and grasping tasks showing the arm's, gripper's and object's frames

\subsection{Visibility and Grasping Task Results}

Experiment photos of the visibility and grasping tasks executed by Nao robot are presented in Fig. 6 and correspond to the tasks introduced in sections 4.2..4.5 above. Fig. 6-a, shows the item to grasp before launching the MBT to detect and track it (Fig. 6-b). The visibility task is used to center the object on the camera's image, and the pre-grasping task is executed in Fig. 6-c, where we can identify the different frames on the robot's arm and gripper in addition to the object's frame. Afterwards, the gripper's frame approaches the object's when executing the grasping task (Fig. 6-d). Finally the gripper closes and the manipulation task is completed (Fig. 6-e-f).

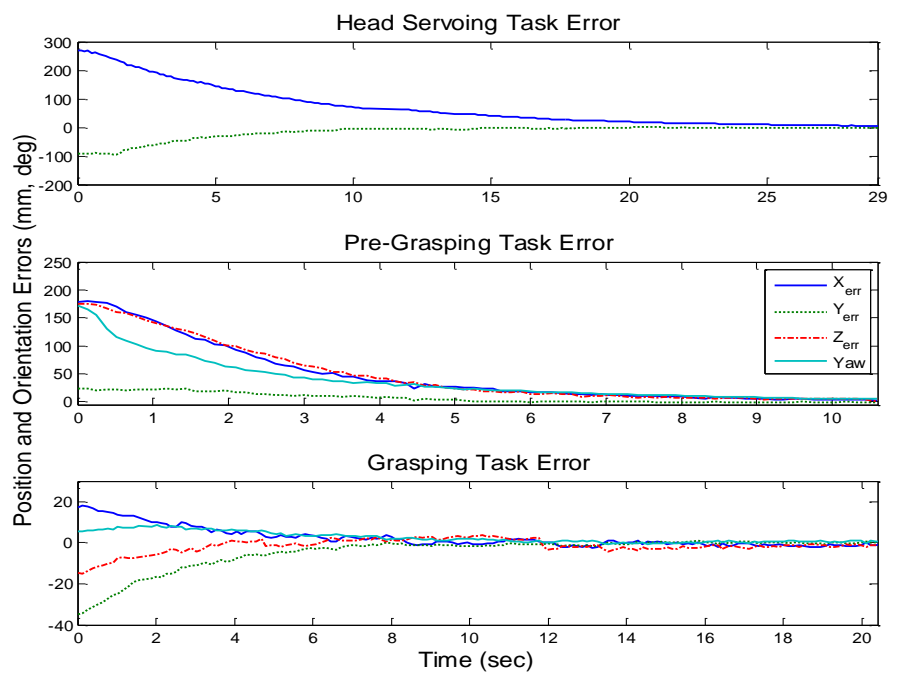

Fig. 7 Experimental results: Visibility task, Pre-Grasping task and Grasping task errors 
The variation of the error in each task is presented in Fig. 7: the first graph represents the horizontal and vertical position error during the head servoing task (visibility task), initially the object is at a distance of approximately $30 \mathrm{~cm}$ from the center of the camera's image, we remark that this error is successfully regulated to zero during $29 \mathrm{sec}$ with a precision of $5 \mathrm{~mm}$.

For the $2^{\text {nd }}$ and $3^{\text {rd }}$ graphs, we represent the variation of pre-grasping and grasping tasks errors on $\mathrm{X}, \mathrm{Y}$ and $\mathrm{Z}$ components (in Nao's frame), and the Yaw angle of the gripper orientation: the robot's hand is initially at an approximate distance of $25 \mathrm{~cm}$ from the predefined pre-grasping position, and the gripper is rotated of $180 \mathrm{deg}$ with respect to the object. During this task, the position error and the Yaw angle are regulated exponentially to zero during $31 \mathrm{sec}$. Finally, we recall that these (pre) grasping tasks are successfully executed with a precision of $5 \mathrm{~mm}$ and $1 \mathrm{~mm}$ respectively and $3 \mathrm{deg}$ for the orientation.

To ensure the robustness of the proposed visual servoing technique for manipulation tasks and the efficiency of the used control laws. The experiment on the previously presented tasks (tracking, head servoing and object grasping) have been successfully repeated 40 times with the same initial conditions. The average of the required convergence time for each task has been also calculated: $26.3 \mathrm{sec}$ for visibility task and $28.4 \mathrm{sec}$ for object manipulation task.

\section{Discussions and Conclusions}

In this study, we presented a concrete scenario of a humanoid mobile robot executing self-localization task while walking and manipulation tasks in an everyday life environment, and we detailed the elementary tasks used by the robot to perform this scenario. The experimental results point out the possibility and efficiency of using the MBT techniques to apply real-time 3D visual servoing on a simple humanoid robot (Nao) in case of localization and manipulation tasks.

For the self-localization task, the error may be relatively large with respect to other localization methods, but it is acceptable and sufficient when dealing with indoor locomotion for manipulation tasks, because of the high robustness of the MBT technique and the implemented automatic re-initialization of the tracking process. In Fig. 5, between the $10^{\text {th }}$ and $32^{\text {nd }}$ sec, we can identify some disturbances which are caused by the displacement of the camera during the robot's locomotion. During this period, the automatic re-initialization module of the tracking is used to prevent localization task from failure. To improve this method, an additional module could be used to dynamically compensate the camera's motions during robot's walking (using data from robot's accelerometer/gyrometer), or by fusion with other sensors data; for example using an external camera on the ceiling of the room to provide a wider view and more visual data, or the Nao's odometry module. 
For the manipulation part, the results of the tracking and grasping tasks, which were tested several times, show that this method is robust to camera occlusion by the robot's hand, and robust to slight object movement due to hand-object collision. These tasks should be improved and tested on other robots. Indeed, the mechanic and software architecture of Nao is very constraining, especially for the small camera field of view, the inability of simultaneous use of the two cameras and the constrained operational space of the hands. For this, future works will concentrate on the improvement and implementation of this method on other platforms with different objects of complex shapes with mobile or articulated elements. Other sensors' feedbacks can also be used to improve the manipulation robustness and reactivity against dynamic or unusual changes in the environment.

\section{References}

1. Brock O, Kuffner J, Xiao J (2008) Motion for manipulation tasks. In: Springer Handbook of Robotics, Siciliano B and Khatib O (ed) Springer Berlin Heidelberg, pp. 615-645.

2. Evrard P, Mansard N, Stasse O, Kheddar A, Schau T, Weber C, Peer A, Buss M (2009) Intercontinental, multimodal, wide-range telecooperation using a humanoid robot. In: IEEE/RSJ Int. Conf. on Intelligent Robots and Systems - IROS'09, pp. 5635-5640.

3. Nitzsche N, Schmidt G (2004) A mobile haptic interface mastering a mobile teleoperator. In: IEEE/RSJ Int. Conf. on Intelligent Robots and Systems - IROS'04, vol. 4, sept-oct. 2004, pp. 3912-3917.

4. Houston K, Sieber A, Eder C, Vittorio O, Menciassi A, Dario P (2011) A teleoperation system with novel haptic device for micromanipulation. In: Int. Journal of Robotics and Automation, vol. 26, no. 3, 2011.

5. Miller W T (1987) Sensor-based control of robotic manipulators using a general learning algorithm. In: IEEE Journal of Robotics and Automation, vol. 3, no. 2, pp. 157-165, apr. 1987.

6. Dang H, Allen P (2010) Robot learning of everyday object manipulations via human demonstration. In: IEEE/RSJ Int. Conf. on Intelligent Robots and Systems - IROS'10, oct. 2010, pp. 1284-1289.

7. Jain A, Kemp C (2009) Pulling open novel doors and drawers with equilibrium point control. In: IEEE-RAS Int. Conf. on Humanoid Robots, dec. 2009, pp. 498-505.

8. Mansard N, Stasse O, Chaumette F, Yokoi K (2007) Visually-guided grasping while walking on a humanoid robot. In: IEEE Int. Conf. on Robotics and Automation, apr. 2007, pp. 3041-3047.

9. Quinlan M J, Middleton R H (2009) Multiple model kalman filters: A localization technique for robocup soccer. In: RoboCup 2009: Robot Soccer World Cup XII, Baltes J, Lagoudakis M G, Naruse T, Ghidary S S (ed) Springer, 2010, vol. 5949, pp. 276-287.

10. Billington D, Estivill-Castro V, Hexel R, Rock A (2006) Using temporal consistency to improve robot localisation. In: RoboCup 2006: Robot Soccer World Cup X, Lakemeyer G, Sklar E, Sorrenti D, Takahashi T (ed) Springer Berlin/Heidelberg, 2007, vol. 4434, pp. 232-244.

11. Haverinen J, Kemppainen A (2009) Global indoor self-localization based on the ambient magnetic field. In: Int. Journal of Robotics and Autonomous Systems, vol. 57, no. 10, pp. 1028-1035, oct. 2009.

12. Thuilot B, Martinet P., Cordesses L, Gallice J (2002) Position based visual servoing: keeping the object in the field of vision. In: IEEE Int. Conf. on Robotics and Automation, vol. 2, 2002. 
13. Gouaillier D, Hugel V, Blazevic P, Kilner C, Monceaux J, Lafourcade P, Marnier B et al (2009) Mechatronic design of Nao humanoid. In: IEEE Int. Conf. on Robotics and Automation, may 2009, pp. 769-774.

14. Chaumette F, Hutchinson S (2006) Visual servo control. i. basic approaches. In: IEEE Robotics Automation Magazine, vol. 13, no. 4, pp. 82-90, dec. 2006.

15. Cervera E (2006) A Cross-Platform Network-Ready Visual Servo Simulator. In : IEEE Int. Conf. on Intelligent Robots and Systems (IROS'06), pp. 2314-2319, Beijing (China), 2006.

16. Marchand E, Spindler F, Chaumette F (2005) Visp for visual servoing: a generic software platform with a wide class of robot control skills. In: IEEE Robotics Automation Magazine, vol. 12, pp. 40-52, dec. 2005.

17. Comport A, Marchand E, Pressigout M, Chaumette F (2006) Real-time markerless tracking for augmented reality: the virtual visual servoing framework. In: IEEE Transactions on Visualization and Computer Graphics, vol. 12, no. 4, pp. 615-628, jul-aug. 2006.

18. Galindo C, Fernandez-Madrigal J-A, Gonzalez J, Saffiotti A (2008) Robot task planning using semantic maps. In: Robotics and Autonomous Systems, vol. 56, no. 11, pp. 955-966, 2008.

19. Stasse O, Verrelst B, Vanderborght B, Yokoi K (2009) Strategies for humanoid robots to dynamically walk over large obstacles. In: IEEE Transactions on Robotics, vol. 25, no. 4, pp. 960-967, aug. 2009.

20. Sorribes J, Prats M, Morales A (2007) Visual tracking of a jaw gripper based on articulated 3d models for grasping. In: IEEE Int. Conf. on Robotics and Automation, may 2010, pp. 2302-2307. 